\title{
Influencia de los componentes minoritarios de los carbones en la fabricación de cementos( $\left(^{*}\right)$
}

FRANCISCO SORIA SANTAMARIA, Dr. en Quimica Industrial Profesor de Investigación del I.E.T.C.C. - C.S.I.c.

\author{
$R E S U M E N$ \\ Como consecuencia de la reconversion al carbón \\ en la industria cementera, se considera importante \\ analizar la incorporación y trascendencia de \\ ciertos componentes en el proceso de clinkerización. \\ Se expone con detalle los circuitos de gases \\ $y$ materias volátiles en el proceso de cocción, \\ destacando el interés del azufre, àlcalis, cloruros \\ $y$ fluoruros. \\ Se destacan las condiciones y limites impuestos \\ por la práctica a ciertos componentes y las \\ posibilidades de aplicación de los carbones en este \\ sentido. \\ Finalmente, se hace un breve comentario a la \\ emision de polvo y gases contaminantes, \\ precisando la incidencia en este campo con el \\ empleo de combustibles sólidos en los hornos para \\ fabricar cemento.
}

\author{
$S U M M A R Y$
}

As a consequence of the reconversion of coal in the cement industry, it is considered important to analyze the incorporation and transcendence of certain components in the clinkerisation process.

Given in detail are the circuits of gases and volatile materials used in the baking process, emphasizing the interest of sulphur, alkalis, chlorides and fluorides.

Emphasized are the conditions and limits imposed in practice to certain components and the possibilities of application of the carbons in this sense.

Finally, a brief comment is made to the emission of dusts and contaminating gases, emphasizing the incidence in this field with the use of solid fuels in the furnaces to manufacture cement.

\section{INTRODUCCION}

La fabricación del clínker de cemento portland (base de todos los conglomerantes silicocalcáreos), lleva consigo la cocción de un producto finamente molido (100\% inferior a 150 micras y del orden del $50 \%$, siempre en peso, por debajo de 45 micras, con una superficie específica de $5.000-6.000 \mathrm{~cm}^{2} / \mathrm{g}$ expresada en unidades Blaine).

La composición básica de este producto procede de rocas naturales (calizas, margas, arcillas, pizarras), con la adición circuntancial de correctores, principalmente silíceos y ferruginosos. Algo más de las tres cuartas partes lo constituye el carbonato cálcico y el resto materiales silicoaluminosos, acompañados de otros componentes que, como los álcalis, la magnesia, el azufre (generalmente en forma de sulfatos), el titanio, el manganeso, etc., se encuentran con carácter minoritario.

Expresada en forma de óxidos, como es normal en la terminología cementera, la composi-

* Conferencia pronunciada en el Coloquio-Panel sobre "El futuro de los carbones españoles" celebrado en León los días 16, 17 y 18 de septiembre de 1982. 
ción del crudo (desprovisto ya de la pérdida por calcinación), oscila entre los siguientes lí mites:

\begin{tabular}{|c|c|}
\hline $\begin{array}{ccccccccccc}\mathrm{CaO} & \ldots & \ldots & \ldots & \ldots & \ldots & \ldots & \ldots & \ldots & \ldots & \ldots\end{array}$ & $60-66 \%$ \\
\hline 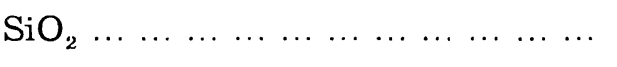 & $18-23$ \\
\hline 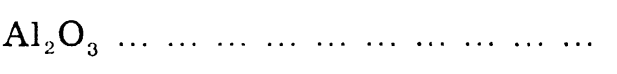 & 3- 6 \\
\hline 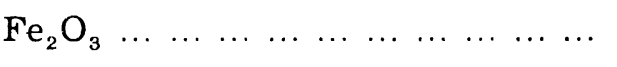 & \\
\hline Eompon & \\
\hline
\end{tabular}

El crudo se calienta progresivamente hasta una temperatura de alrededor de $1.450^{\circ} \mathrm{C}$ por los gases procedentes de la combustión de carbón, fuel-oil o gas. El material fluye en contracorriente con los gases calientes en un horno que, esencialmente, consta de un cilindro girando lentamente sobre su eje y ligeramente inclinado. En el extremo inferior se inyecta el combustible (total o parcialmente), en tanto que el crudo se alimenta por su parte superior.

El calor residual de los gases de escape se recupera, en lo posible, en otros equipos de la instalación: en los procesos seco y semiseco, el intercambio térmico se concluye fuera del horno tubular para secar, precalentar y precalcinar, en parte, el crudo en intercambiadores de lecho poroso (parrilla del proceso semiseco) o en intercambiadores de ciclón (proceso seco); en el sistema de vía húmeda (prácticamente excluido hoy día por su elevado consumo térmico), el intercambio de calor para realizar las operaciones antes mencionadas tiene lugar en la primera parte del propio tubo del horno, empleando los sistemas mecánicos adecuados.

Dentro del sistema de cocción se establecen zonas, donde tienen lugar tres grupos principales de reacciones:

- deshidratación, con pérdida de agua libre y agua combinada, hasta una temperatura de unos $500^{\circ} \mathrm{C}$.

- descarbonatación, que lleva consigo la eliminación de $\mathrm{CO}_{2}$, por descomposición de los carbonatos de calcio y magnesio, de 600 a $1.000^{\circ} \mathrm{C}$.

- clinkerización, con la formación de compuestos con propiedades hidráulicas $\left(1.000-1.450^{\circ} \mathrm{C}\right)$ y la aparición de una fase líquida fundida $\left(1.300-1.450^{\circ} \mathrm{C}\right)$.

\section{COMBUSTIBLES EMPLEAdOS EN LA FABRICACION DE CEMENTO}

La primera fuente de energía térmica en los hornos rotatorios para fabricar cemento fue el carbón. Esto sucedía en las postrimerías del siglo pasado. A partir de 1949 se emplearon el fuel-oil pesado y los gases (de cockería y natural). En 1950 se conocía la combustión mixta fuel-oil/carbón y, poco más tarde, la mixta carbón/gas.

Entre 1960 y 1970 muchas instalaciones funcionaron con combustión mixta fuel-oil/carbón, con tendencia a fuel-oil como único combustible, que llegó a ser mayoritario (del 75 a casi el $100 \%$ ) en el consumo total de energía primaria, hasta finales de los años 70, en los paises desarrollados de la Europa Occidental y Japón. Algunos de ellos, como la República Federal Alemana y Francia, incrementaron paralelamente el consumo de gas natural en este último período. Los EE.UU. de América fueron más conservadores en la evolución, 
sin llegar nunca a bajar del $40 \%$ de carbón en el consumo total de energía térmica para la industria del cemento.

Los años 80,81 y 82 pueden considerarse como años de transición con un fuerte incremento en el consumo de carbón y un retroceso brusco en el del fuel-oil. Es decir, el cruce de porcentajes de consumo de carbón y fuel-oil en los años 60-73, se repite en sentido contrario en los años 78-82, pero en un plazo de tiempo mucho más corto. En la figura 1 se representa la evolución del consumo de combustibles en la industria cementera francesa desde 1960, evolución que, en términos generales, es similar a la experimentada por otros países de la Europa Occidental, incluida España.

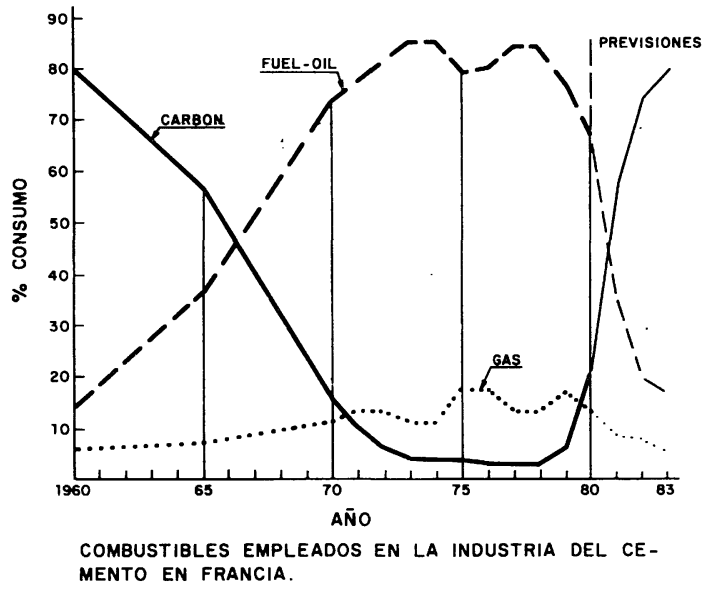

Fig. 1.-Combustibes empleados en la industria de cemento en Francia.

\subsection{Componentes menores en los combustibles}

El gas natural está prácticamente exento de áicalis y de compuestos de cloro. Puede contener cantidades, incluso considerables, de $\mathrm{SH}_{2}$ pero, en este caso, se desulfura antes de emplearlo como combustible.

El fuel-oil empleado en las fábricas de cemento (fuel-oil pesado) contiene pequeñas cantidades de cenizas, que pueden oscilar de 0,01 a $0,1 \%$ en peso, según su origen, y cuyos componentes principales son $\mathrm{SiO}_{2}, \mathrm{Al}_{2} \mathrm{O}_{3}, \mathrm{~K}_{2} \mathrm{O}, \mathrm{Na}_{2} \mathrm{O}$, y pudiéndose encontrar metales pesados como $\mathrm{Ni}, \mathrm{V}, \mathrm{Pb}$ y $\mathrm{Zn}$ en concentraciones en el entorno de milésimas \% (1).

El contenido medio en $\mathrm{Cl}^{1-}$ es de $0,05 \%$ (2) y el de azufre oscila de 2,5 a 5,0 \% (3), ambos en peso.

Los carbones, en general, son más variables en su composición, dependiendo de las circunstancias en que se han formado.

Una característica importante son las cenizas, cuyo contenido puede variar entre 3 y $30 \%$, aproximadamente. En su composición se encuentran minerales de la arcilla, cuarzo, pirita y minerales carbonatados (sobre todo en algunos lignitos). Las cenizas ácidas pueden tener tendencia a formar masas fundidas de baja viscosidad que reaccionan en el horno con el crudo en proceso de cocción, facilitando la tendencia a formar costras y anillos (4).

La presencia de cenizas obliga a modificar la composición del crudo para mantener el factor de saturación en cal, siendo importante su homogeneidad de mezcla con la carga del horno, lo cual se logra bastante bien si el carbón se emplea como combustible en los nuevos sistemas de precalcinación. En este caso, se pueden aplicar carbones muy pobres sin alterar la calidad del clínker (5).

Una influencia muy particular en el proceso de cocción es la ejercida por el azufre, los cloruros y los álcalis.

En los carbones, el contenido de álcalis no pasa, en principio, de $0,1 \%$ en peso, para un contenido en cenizas inferior al $20 \%$. Estos álcalis lo mismo que los del crudo (que pue- 
den alcanzar casi el $2 \%$ sobre clínker) tienen mucha influencia sobre la marcha del proceso y sobre la emisión de $\mathrm{SO}_{2}$ a la atmósfera, como se matizará más adelante.

El contenido de cloruros en los carbones varía de 0,10 a $0,15 \%$ en peso, con excepciones que, para los carbones europeos alcanzan el $0,25 \%$ y para algunos carbones norteamericanos el $0,35 \%$ en peso (2). En carbones particularmente ricos en cenizas se han medido contenidos en cloruros de hasta $0,4 \%$ en peso.

Es de señalar que una incorporación grande de cloruros en el horno facilita la formación de costras y anillos aparte de otras perturbaciones (6).

El azufre se encuentra en los carbones en forma de pirita, marcasita y, en más pequeña cantidad, de sulfatos (alcalinos o alcalinotérreos) y de azufre orgánico, en contenidos totales que varían, en principio, entre 0,5 y $1,2 \%$ en peso de $\mathrm{S}$, con valores extremos superiores al $2 \%$ en carbones de calidad inferior (pizarras bituminosas, por ejemplo).

Los carbones contienen también trazas de elementos tales como $\mathrm{Pb}, \mathrm{Zn}, \mathrm{Ni}, \mathrm{V}, \mathrm{Co}$, As y $\mathrm{Ti}$, que se valoran en partes por millón.

\section{CIRCUITOS DE GASES Y MATERIALES VOLATILES EN EL PROCESO DE COCCION}

La corriente gaseosa, que fluye en sentido contrario a la corriente del crudo en proceso de cocción, contiene, además del polvo de dicho producto, combinaciones de álcalis, azufre, cloro y fluor. Estas se han formado, principalmente, a partir de compuestos vaporizados y disociados de la carga del horno y del combustible, como resultante, en gran parte, de reacciones que tienen lugar en la atmósfera gaseosa.

Si tales compuestos reaccionan con el producto de la cocción o se precipitan sobre él en las partes frías del horno, en el intercambiador o en las instalaciones de secado conectadas al proceso de cocción, se forma lo que se llama un "circuito interno".

$\mathrm{Si}$, por el contrario, se depositan en las instalaciones de depuración de gases y se vuelven al horno, adicionándolos en cualquier punto del proceso de materias primas, se origina el llamado "circuito externo".

Si los productos depurados se retiran del proceso o se descargan a la atmósfera con los gases depurados, no tiene lugar ningún circuito o proceso cíclico.

Como resultado de los procesos cíclicos, la concentración de los constituyentes citados aumenta en el producto en trance de cocción y, en consecuencia, en el propio clínker, hasta límites, según el caso, definidos por las propias características de los compuestos formados. Para cada uno de ellos, el equilibrio se alcanzará cuando las cantidades que entran con la alimentación, polvo recuperado y combustible son iguales a las que salen por el clínker, gases de escape y polvo eliminado.

La acumulación de estos compuestos en la carga puede modificar el comportamiento de ésta en el horno, singularmente sus propiedades fundentes; además, cabe la posibilidad de una reacción directa entre los constituyentes de los gases del horno y el producto sólido. Todo ello puede dar lugar a la formación de depósitos y anillos.

Estas formaciones, en principio, no se relacionan con las procedentes de las cenizas de los carbones (ya que se forman también con combustibles exentos de cenizas), aunque sí tengan sus concomitancias. 
Lógicamente, estas reacciones en la atmósfera gaseosa o las que tienen lugar entre los gases y el sólido influyen de una forma decisiva en la emisión de gases del sistema: cuanto mayor sea la proporción que permanece en el material consecuencia de reacciones, tanto menor será la proporción de compuestos gaseosos emitidos.

\subsection{Alcalis}

Como cuestión previa debe recordarse la importancia de su presencia en el clínker, en tres aspectos de las propiedades del cemento: expansión árido-álcali, fraguado y resistencias mecánicas.

Un elevado contenido de álcalis en el cemento puede dar lugar a reacciones de carácter expansivo de consecuencias nefastas para la estabilidad de la obra si se emplean áridos reactivos. Entre los áridos reactivos se encuentran la sílice o silicatos geológicamente jóvenes como ópalo, calcedonia, tridimita y silicatos vitrificados. Por este motivo, para evitar estas reacciones, se limita el contenido total de álcalis en los cementos para ciertas aplicaciones.

También los álcalis pueden afectar al fraguado del cemento si se transforman en carbonatos por efecto del carbónico del aire. Esta influencia no es muy importante si los álcalis se encuentran como sulfatos. A veces, durante el ensilado del cemento, se forman grumos atribuidos a otro sulfato: la singenita $\left(\mathrm{K}_{2} \mathrm{SO}_{4} \cdot \mathrm{CaSO}_{4} \cdot \mathrm{H}_{2} \mathrm{O}\right)$ (7).

Si los álcalis no se encuentran en forma de sulfatos, sino incluidos en silicatos y aluminatos, reducen las posibilidades de saturación en cal y, llegado el caso, puede surgir una mayor proporción de cal libre. Esto afecta notoriamente el desarrollo resistente, sobre todo en su fase inicial.

El contenido de álcalis de los crudos en Europa oscila de 0,5 a $2 \%$ en peso para el $\mathrm{K}_{2} \mathrm{O}$ y de 0,05 a $0,6 \%$ en peso para el $\mathrm{Na}_{2} \mathrm{O}$; predomina el primero en relaciones que oscilan de $1 / 3$ a $1 / 10$.

En las materias primas, los álcalis se encuentran presentes principalmente en los minerales de la arcilla, en la mica y en los feldespatos; las cantidades de álcalis contenidas en la caliza y en los combustibles son, generalmente, poco importantes, aunque, en estos últimos, dependa de su origen y contenido de cenizas como se dijo anteriormente (en las cenizas el contenido de álcalis oscila de 1 a $6 \%$ ).

A las altas temperaturas de la zona de sinterización en el horno rotatorio $\left(>1.350^{\circ} \mathrm{C}\right)$ se descomponen los minerales alcalinos y los álcalis se subliman en forma de óxidos, aunque con más intensidad el potásico que el sódico. Según C. Goes (8), la evaporación comienza en la mica y, en particular en la illita, a temperaturas más bajas que para el feldespato.

Esta volatilidad de los álcalis se facilita:

- Aumentando la temperatura y el tiempo de permanencia en la zona de sinterización (9).

- Empleando combustibles pobres en azufre y materias primas pobres en azufre y en óxido de hierro (el óxido de hierro facilita la formación de fase líquida, se reduce la porosidad de los nódulos y dificulta la difusión).

- Aportando vapor de agua a la llama o recurriendo a combustibles que producen vapor de agua abundante (gas natural, por ejemplo). 
- Empleando materias primas que contengan illita o mica.

- Añadiendo cloruros o fluoruros al material de alimentación (10) (11); precisamente, los cloruros son los compuestos alçalinos, de los posibles, con mayor tensión de vaporización en la cocción del clínker.

Los álcalis vaporizados y disociados reaccionan en las partes más frías del horno, bajo condiciones oxidantes, con el $\mathrm{SO}_{2}, \mathrm{CO}_{2}$ y cloruros presentes también en los gases, dando lugar a sulfatos, carbonatos y cloruros alcalinos.

Los sulfatos se forman y condensan en primer lugar y a temperatura de los gases más elevada; carbonatos y cloruros no se formarán hasta que prácticamente no quede dióxido de azufre disponible y la temperatura de los gases haya descendido por debajo de $1.200^{\circ} \mathrm{C}$ (12).

Los vapores de estos compuestos alcalinos condensan en zonas del horno menos calientes (generalmente sobre el producto en proceso de cocción), en el orden citado de más a menos temperatura (sulfatos, carbonatos y cloruros).

Los compuestos condensados sobre el material entran de nuevo en el horno y establecen así un circuito interno.

Como los sulfatos alcalinos son menos volátiles, prácticamente no sufren posterior vaporización y abandonan el horno juntamente con el clínker. La fracción de sulfatos que, en su vaporización, atraviesa la barrera del precalentador o intercambiador se retiene en el electrofiltro, donde se recupera un polvo cuyo contenido en sulfatos alcalinos y alcalinotérreos puede alcanzar el $30 \%$ en peso, empleando combustibles normales sólidos o líquidos.

Los carbonatos y cloruros alcalinos, más volátiles, vaporizan de nuevo y entran una vez más en el circuito interno.

Se puede formar un circuito externo con los compuestos alcalinos que descargan en el depurador, si el polvo vuelve de nuevo al horno mezclado con el crudo.

\subsection{Azufre}

El azufre se introduce en el proceso de cocción con las materias primas y con el combustible.

En las materias primas se encuentra como sulfatos, generalmente alcalinotérreos, como sulfuros y, a veces, en forma de compuestos orgánicos (presencia de combustible).

En los combustibles sólidos, como ya se dijo, en forma de sulfatos (cálcico generalmente) o sulfuros del hierro.

Como se comentó al hablar de los álcalis, el $\mathrm{SO}_{2}$ formado en el proceso de combustión reacciona, en primer lugar, con los álcalis vaporizados, pero también con los álcalis que aún están presentes en forma sólida en la carga del material. Como resultado de estas reacciones se forman sulfatos alcalinos.

De los estudios llevados a cabo por S. Sprung (13) y H. Ritzmann (14) el dióxido de azufre, si no hay suficientes álcalis para combinar con él, combinará además en forma de sulfato cálcico, y en presencia de oxígeno, en primer lugar, con la cal $\mathrm{CaO}$ formada en la calcinación $\mathrm{y}$, posteriormente, con el carbonato cálcico del crudo. 
La reacción con el $\mathrm{CaO}$ tiene lugar, principalmente, en el horno, a temperaturas relativamente altas; la reacción con el carbonato cálcico ocurre también en el intercambiador y, particularmente, en la planta de secado y molienda conjuntos y torres de enfriamiento, a temperaturas inferiores a $400^{\circ} \mathrm{C}$, donde la reacción se facilita por el estrecho contacto con las nuevas superficies creadas en la molturación (15).

$\mathrm{El} \mathrm{SO}_{2}$ puede también combinar con silicatos y aluminatos para formar compuestos tales como $\left(2 \mathrm{CaO} \cdot \mathrm{SiO}_{2}\right) \cdot \mathrm{CaSO}{ }_{4}$ y $\mathrm{C}_{3} \mathrm{~A} \cdot \mathrm{CaSO}_{4}$, que descomponen, posteriormente, en la zona de clinkerización.

\subsection{Cloruros}

El contenido en cloruros de las materias primas suele ser bajo, aunque, a veces, excede del $0,3 \%$. El carbón, en general, contiene como ya se dijo (2) entre 0,01 y $0,15 \%$ en peso, aunque en combustibles ricos en cenizas puede llegar a $0,4 \%$.

Los cloruros liberados durante el calentamiento del crudo y la combustión reaccionan con los álcalis, o bien en la carga de material, o bien en los gases del horno después de la vaporización, dando lugar a cloruros alcalinos.

Estos cloruros condensan sobre el material en zonas más frías (por debajo de $800^{\circ} \mathrm{C}$ ), particularmente, a la entrada del horno y en el precalentador o inercambiador. El circuito interno de cloruros es grande y su acumulación crea serias perturbaciones en el proceso.

Por esta razón, los hornos de vía seca y, en particular, los que disponen de precalentador en suspensión, exigen combustibles (y, por supuesto, materias primas) con un contenido en cloruros muy limitado.

La eliminación de polvo del electrofiltro, suprimiendo el circuito externo, polvo que en estos hornos es siempre más bien pobre en compuestos volátiles (19), no permite reducir el circuito interno (3). Entonces para conseguir esta reducción, por encima de un cierto límite del total de cloruros introducidos en el proceso, es necesario desvíar (bypass) una fracción de los gases del horno antes del electrofiltro, tanto mayor cuanto más elevada sea la aportación de cloruros (20) (Figura 2).

\subsection{Fluoruros}

El contenido en fluoruros de los crudos osci-

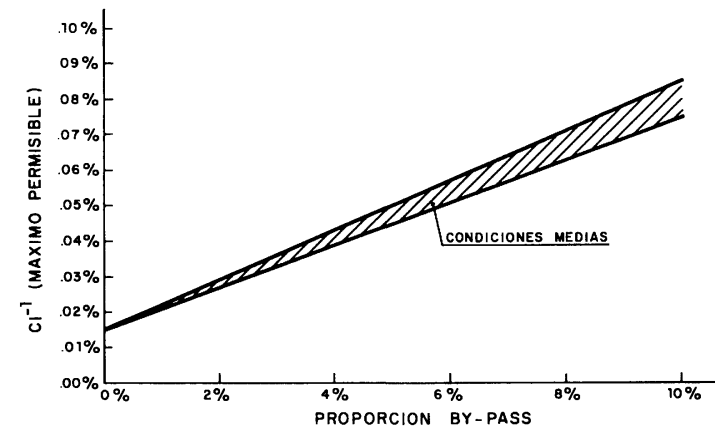

HORNO CON PRECALENTADOR EN CUATRO ETAPAS PORCENTAJE MAXIMO DE CLORUROS PERMISIBLE VERSUS PROPORCION DE BY-PASS.

Fig. 2.-Horno con precalentador en cuatro etapas.

Porcentaje máximo de cloruros permisible versus proporción de by-pass. la entre 0,02 y $0,07 \%$; los carbones contienen hasta $0,02 \%$ y el fuel-oil hasta $0,002 \%$, siempre en peso (23).

Participan en los procesos cíclicos del horno de cemento de la misma manera, más o menos, que los demás constituyentes volátiles.

Por los estudios citados (23), del 88 al $98 \%$ de los fluoruros introducidos en el horno descargan con el clínker, proporción tan alta que hace que el circuito interno correspondiente no dé lugar a acumulaciones perturbadoras ni otros desórdenes operacionales. El resto del fluor descarga en el polvo del electrofiltro, sin llegar a producirse la emisión de compuestos de fluor con los gases del horno. 
Lógicamente, el volumen de los ciclos o circuitos de los componentes citados no depende sólo de su proporción en las materias primas y en los combustibles. También depende del tipo de horno: el volumen de los ciclos aumentará al reducirse la longitud del horno y al pasar de gránulos a polvo la forma de alimentar el crudo, debido a:

- una volatilización más intensa en material pulverulento que granular;

- mayores temperaturas en la zona de clinkerización de hornos cortos como son los de vía seca con intercambiador en suspensión gaseosa.

\section{CONDICIONES Y LIMITES IMPUESTOS POR LA PRACTICA A LOS COMPONENTES DE LOS CIRCUITOS DE GASES Y PRODUCTOS VOLATILES}

Según B. Osbaeck (16) y R. Bucchi (3), las condiciones más favorables para el desarrollo del proceso de cocción, en lo que se refiere al azufre, se logran al conseguir un valor del "módulo de sulfatos corregido" comprendido entre 0,83 y 0,95

$$
0,83 \leqslant \mathrm{M}^{*} \mathrm{SO}_{3}=\frac{\left[\mathrm{SO}_{3}\right]}{\left[\mathrm{K}_{2} \mathrm{O}\right]+\left[\mathrm{Na}_{2} \mathrm{O}\right]-\left[\mathrm{Cl}^{1-}\right]} \leqslant 0,95 \mathrm{~mol} \cdot \mathrm{mol}
$$

donde los valores entre corchetes representan concentraciones molares, referidas al clínker, de las especies químicas introducidas en el proceso con el crudo y con el combustible.

Para $\mathrm{M}^{*} \mathrm{SO}_{3}>0,95$, aumenta la tendencia a la formación de costras y anillos de $2 \mathrm{C}_{2} \mathrm{~S}$. . $\mathrm{CaSO}_{4}$ (sulfoespurrita) y de $\mathrm{C}_{3} \mathrm{~A} \cdot \mathrm{CaSO}_{4}$ (sulfoaluminato de calcio); los gases de escape contienen $\mathrm{SO}_{2}$.

Para $\mathrm{M}^{*} \mathrm{SO}_{3}<0,83$, los álcalis no combinados con el azufre se combinarán con los minerales del clínker y aumentarán la intensidad del circuito interior; los gases de escape sólo contienen trazas de $\mathrm{SO}_{2}$.

Para darse una idea del contenido en azufre del combustible que satisfaga la ecuación antes señalada, se pueden despreciar los álcalis del combustible y los cloruros de crudo y combustible, considerando como valores medios de $\mathrm{Na}_{2} \mathrm{O}, \mathrm{K}_{2} \mathrm{O}$ y $\mathrm{SO}_{3}$ presentes en el crudo ("crudo medio"), $0,18 \%, 0,56 \%$, y $0,27 \%$ en peso, respectivamente (3). Con estos valores del crudo, $\mathrm{M}^{*} \mathrm{SO}_{3}=0,38 \mathrm{~mol} \cdot \mathrm{mol}^{-1}$.

La ecuación citada toma el valor de $0,9 \mathrm{~mol} \cdot \mathrm{mol}^{-1}$ (media entre 0,83 y 0,95 ) cuando la aportación de $\mathrm{SO}_{3}$ con el combustible equivale a $0,59 \%$ en peso referido al clínker, es decir, cuando el contenido en $\mathrm{S}$ del combustible (porcentaje en peso sobre combustible exento de humedad) es:

$$
\mathrm{S}^{*}=\mathrm{v} \frac{\mathrm{QN}}{x}(\% \text { en peso })
$$

donde QN es el poder calorífico inferior del combustible $\left(\mathrm{kcal} \cdot \mathrm{kg}^{-1}\right)$ y $x$ la demanda específica de energía primaria del proceso de cocción $\left(\mathrm{kcal} \cdot \mathrm{kg}^{-1}\right.$ clínker).

La constante $\mathrm{v}$ vale $0,236 \mathrm{~kg} \cdot \mathrm{kg}^{-1}$ para el "crudo medio" y toma valores diferentes para crudos con distintos contenidos de $\mathrm{Na}_{2} \mathrm{O}, \mathrm{K}_{2} \mathrm{O}$ y $\mathrm{SO}_{3}$. Es de señalar que el "crudo medio" exige un contenido en azufre para el fuel-oil (con demandas específicas de energía de 760$800 \mathrm{kcal} \cdot \mathrm{kg}^{-1}$ clínker) del orden del $3 \%$ (ver figura 3), lo que corresponde con lo que es habitual en la práctica.

Con un carbón de $6.000 \mathrm{kcal} \cdot \mathrm{kg}^{-1}$, para el "crudo medio" y una demanda de energía de $800 \mathrm{kcal} \cdot \mathrm{kg}^{-1}$ clínker se consigue un $\mathrm{M}^{*} \mathrm{SO}_{3} \ldots . .9$. cuando el carbón contiene $1.77 \%$ en 
peso de azufre; en las mismas condiciones, a un lignito de $4.500 \mathrm{kcal} \cdot \mathrm{kg}^{-1}$ se le exigiría un contenido de $\mathrm{S}$ de $1,25 \%$ (ver figura 3 ).

Con estos datos se ve que el problema de un exceso de azufre en los carbones destinados a la cocción del clínker no es frecuente (salvo en el caso de materias primas muy pobres en álcalis y/o muy ricas en sulfatos). El caso contrario es más común, al menos pensando en materias primas españolas.

De todas las maneras, el desequilibrio álcalis/azufre siempre puede ajustarse: en el primer caso, empleando un componente del crudo más rico en álcalis o añadiendo carbonato sódico al crudo; en el segundo, incorporando en el crudo (o en el carbón) pirita, yeso o anhidrita (6) (17) (18).

El límite de cloruros introducidos en el proceso se fija habitualmente (20), (21) (6) en $0,2 \times 10^{-3}-0,25 \times 10^{-3} \mathrm{~kg} \mathrm{Cl}^{1^{-}} \cdot \mathrm{kg}^{-1}$ clínker (120-150 ppm en peso referidas al crudo), pero se reduce a menos de $50 \mathrm{ppm}$ en peso de $\mathrm{Cl}^{1-}$ referidas al crudo, si éste es particularmente rico en $\mathrm{K}_{2} \mathrm{O}$ (17) (22).

Para obtener con los carbones estas condiciones, es preciso que su contenido en $\mathrm{Cl}^{1-}$ sea:

$$
\mathrm{C}^{*} \mathrm{Cl}^{-1} \leqslant \frac{\mathrm{QN}}{40.612} 0,5 \times 10^{-1}(\% \text { en peso })
$$

Se trata de un límite muy estricto para los carbones normales, cuyo contenido en cloruros suele ser superior al del fuel-oil o gas; en algunos casos, puede hacer crítico el proceso de cocción de crudos que no estén prácticamente exentos de cloruros, en hornos con precalentador en suspensión gaseosa.

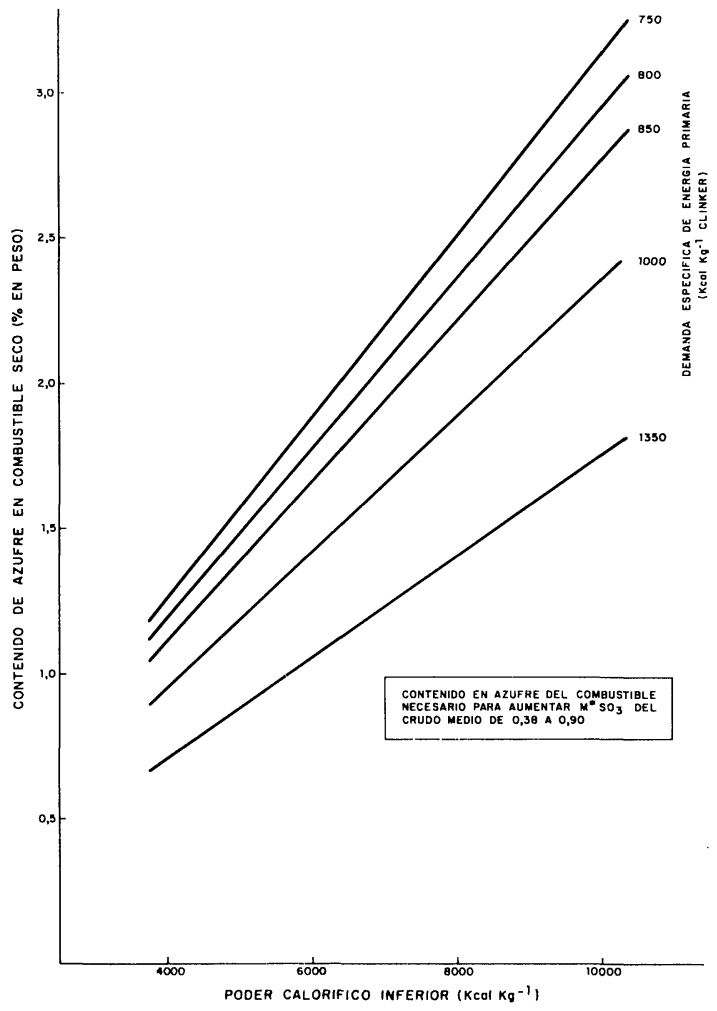

Seguin R. Bucch, y R Bott"

Fig. 3

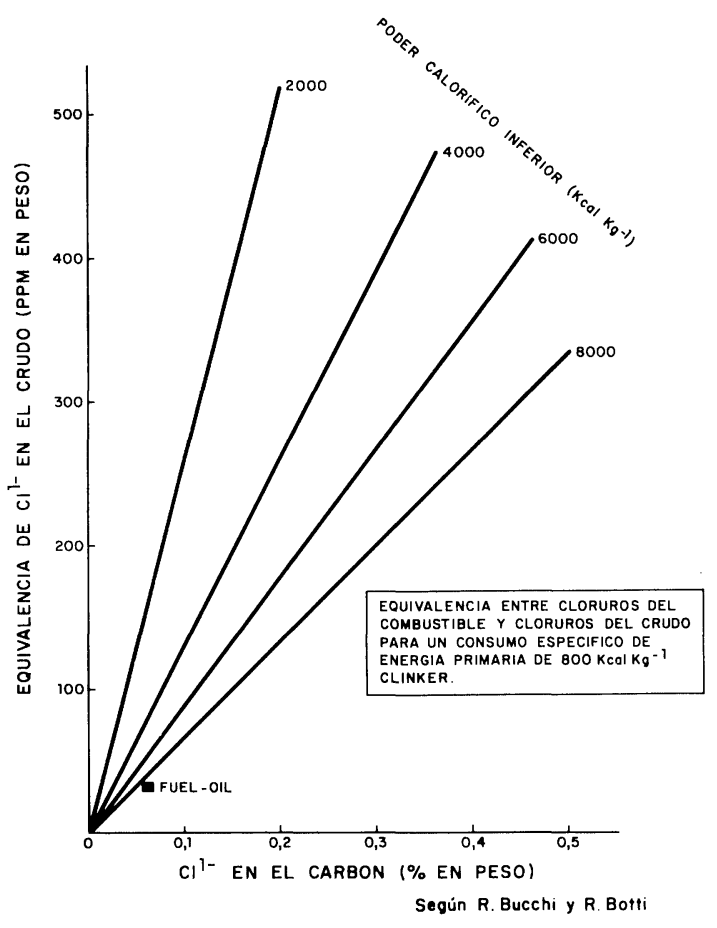

Fig. 4 
El gráfico de la figura 4 permite transformar la concentración de $\mathrm{Cl}^{1-}$ de un combustible de poder calorífico inferior QN, dado en términos de concentración en el crudo.

\section{PERTURBACIONES EN LA MARCHA DEL PROCESO}

Los fenómenos de condensación de los compuestos volátiles pueden crear acumulaciones locales de sales alcalinas que funden produciendo fases líquidas que aglutinan los productos sólidos. Esto da lugar o depósitos a costras, preferentemente en la entrada del horno, escalón inferior de los precalentadores en suspensión y cámara de calcinación en precalentadores de parrilla, es decir, en zonas de la instalación con temperaturas inferiores a $1.100^{\circ} \mathrm{C}$.

Los análisis por técnicas instrumentales de estos depósitos o adherencias muestran que, además de los constituyentes normales del crudo y del clínker, pueden presentarse, entre otros, los siguientes compuestos: (24)

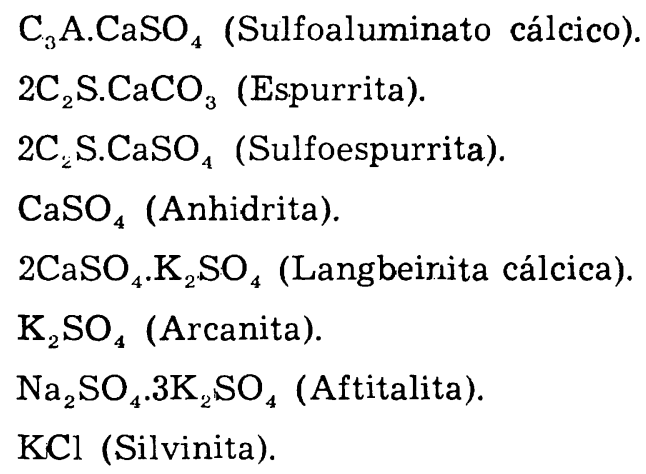

Los compuestos más comunes son los tres últimos sulfatos junto con la silvinita.

La presencia de estos compuestos es inevitable y, a veces, difícil de modificar.

Los álcalis vienen definidos por el tipo de materia prima, y lo mismo podemos decir de los cloruros, que pueden ser aportados también por el combustible, si se emplea carbón.

Sólo es posible jugar con el balance de azufre, que puede alterarse empleando combustibles con contenido variable en este elemento o añadiendo sulfato cálcico al crudo.

El ciclo de álcalis, y, por tanto, su concentración en el proceso a la entrada del horno, puede reducirse si se combina en gran medida con los sulfatos, descargando con el clínker como sulfatos alcalinos. Es decir, puede ser ventajoso aumentar la entrada de azufre (por el combustible o con yeso en el crudo), pero no debe olvidarse que esto puede afectar desfavorablemente las propiedades del clínker ya citadas.

Además, pasados ciertos límites (módulo de sulfatos ya citado) se aumenta la tendencia a formar revestimientos en los precalentadores con la participación de la sulfoespurrita. Incluso, la formación de cantidades sustanciales de $\mathrm{CaSO}_{4}$ (si la participación de sulfatos es aún mayor) puede promover la creación de sales dobles alcalinas-alcalinotérreas y aumentar la tendencia al revestimiento.

En cuanto a los cloruros, como difícilmente descargan con el clínker, se concentran en las partes frías de la instalación e, inevitablemente, crean adherencias, catalizando la formación de espurrita. La tendencia a formar revestimientos crece particularmente si el contenido de cloruros en el crudo es superior a $0,01 \%$ en peso, como ya se ha dicho anteriormente. 


\section{EMISION DE GASES CONTAMINANTES Y POLVO}

\subsection{Polvo}

La industria cementera se ha caracterizado siempre por la manipulación de grandes cantidades de materiales en estado pulverulento. Esto ha conducido a una atención prioritaria a los problemas de depuración, acompañada de una legislación considerada entre las más antiguas y avanzadas del ámbito industrial.

Por eso, el emplear carbón como combustible, no presupone un aumento de emisión de polvo en relación con otros combustibles, dadas las instalaciones de depuración que existen hoy día.

Por otra parte, tal como se desarrolla el proceso en las modernas instalaciones de vía seca, las cenizas del carbón se mezclan íntimamente con el crudo y se comportan como tal crudo en cuanto a las emisiones, que se depuran en el electrofiltro en las mismas condiciones.

Las posibles anomalías en los humos, al emplear carbón como combustible, deben de relacionarse o con una marcha anormal del horno por formación de costras o anillos (debidos, en general al reciclado de cloruros alcalinos) o con una combustión imperfecta.

\subsection{Gases o compuestos contaminantes}

Aunque recientemente se ha hablado de problemas producidos por la emisión de compuestos de talio en alguna fábrica de cemento en la República Federal Alemana (25), esto no puede considerarse como caso general y menos estar relacionado con el combustible empleado en la cocción del clínker.

Aunque los carbones son los únicos combustibles que pueden contener compuestos de talio, sus proporciones son tan bajas que no pueden ser causa única ni prioritaria de posible contaminación. Estas proporciones oscilan de 0,005 a 1,2 ppm de $\mathrm{Tl}_{2} \mathrm{O}$ en peso, lo que representa de $10^{-4}$ a $10^{-1} \mathrm{ppm}$, referidas al clínker.

\subsection{Azufre}

La retención y fijación de azufre del combustible empleado en los modernos hornos de cemento es considerable, dado el fuerte reciclaje y gran intercambio de calor entre gases y material en contracorriente, con el fin de conseguir el mayor ahorro energético posible.

Según H. Ritzman (26) y S. Sprung (13), el azufre retenido en el clínker y en el polvo del electrofiltro es siempre superior al $90 \%$, manteniéndose concentraciones de $\mathrm{SO}_{2}$ en los gases depurados que oscilan de 0 a $480 \mathrm{mg} / \mathrm{m}^{3} \mathrm{~N}$, dependiendo de la relación molar $\mathrm{SO}_{3} / \mathrm{Al}$ calis, es decir, del módulo de sulfatos ya citado.

\subsection{Oxidos de nitrógeno}

Los óxidos de nitrógeno $\left(\mathrm{NO}_{\mathrm{x}}\right)$ se forman en la llama (27):

a) Por fijación térmica del nitrógeno molecular en las zonas de temperatura más elevada.

b) Por conversión (desvolatilización y oxidación) del nitrógeno orgánico en las zonas de temperaturas más bajas. 
En los hornos de cemento, se debe suponer que el $\mathrm{NO}_{\mathrm{x}}$ de fijación térmica es más elevado en la llama de fuel-oil y de gas (prácticamente exento de nitrógeno orgánico) que en la de carbón, al ser mayor con aquéllos la entalpía del aire de combustión.

En cuanto al contenido de nitrógeno orgánico es mayor, en principio, en los carbones que en el fuel-oil (referido a la unidad de energía), pero, el grado de conversión en los carbones no pasa del $25 \%$, en tanto que en el fuel-oil varía de 20 a $90 \%$ (28).

Los resultados de las medidas de R. J. Hilovsky (28) sobre 17 hornos en cinco fábricas de cemento confirman una mayor emisión de $\mathrm{NO}_{\mathrm{x}}$ con fuel-oil que con carbón, demostrando además que el gas natural es, entre ellos, el más contaminante (Tabla 1).

TABLA 1

\begin{tabular}{|l|c|c|c|}
\hline \multirow{2}{*}{ Proceso } & \multirow{2}{*}{ Combustible } & \multicolumn{2}{|c|}{$\mathrm{NO}$ sobre clínker $\left(\mathrm{kg} \cdot \mathrm{t}^{-1}\right)$} \\
\cline { 3 - 4 } & \multirow{2}{*}{ gas } & Intervalo & Valor medio \\
\hline seco & & $7,7-11,2$ & 9,3 \\
húmedo & fuel-oil & $2,3-13,1$ & 5,2 \\
seco & & $1,2-7,3$ & 3,2 \\
húmedo & carbón & $1,6-5,5$ & 2,7 \\
seco & & $1,0-4,4$ & 2,8 \\
\hline
\end{tabular}

El oxígeno afecta a la formación de $\mathrm{SO}_{2}$ y $\mathrm{NO}_{x}$ de modo opuesto: un aumento de su concentración a la entrada del horno reduce el contenido de $\mathrm{SO}_{2}$ en los gases de escape (13) y aumenta el de los óxidos de nitrógeno. En la práctica, puede manejarse como regla empírica que, "en cualquier planta que emplea el mismo combustible, la suma de las emisiones de $\mathrm{SO}_{2}$ y $\mathrm{NO}_{x}$ son siempre prácticamente constantes".

\section{RESUMEN Y CONCLUSIONES}

Hoy en día, teniendo en cuenta las condiciones en que se desarrolla el proceso de cocción en las modernas instalaciones de fabricación de cemento, deben de considerarse los componentes minoritarios de los combustibles.

Las mayores temperaturas de trabajo, el intercambio térmico más perfecto entre gases y sólidos, la recuperación y reciclado de polvo y las mayores exigencias en cuanto a la polución atmosférica se refiere, han hecho surgir problemas nuevos en el proceso y en la calidad del producto obtenido, motivados por la presencia de ciertos componentes, tanto en los crudos como en los combustibles.

Entre estos componentes destacan: los álcalis - mayoritariamente presentes en los crudos-, los sulfatos - normalmente aportados en mayor cuantía por los combustibles-, y los cloruros - que pueden ser objeto de consideración para algunos combustibles, especialmente carbones-. La presencia de otros como fluoruros o componentes metálicos variados, en proporciones generalmente muy minoritarios, no ofrecen, hoy por hoy, especial consideración.

Los álcalis y sulfatos deben mantener cierta relación de proporciones para el mejor desarrollo del proceso. Su aportación total excesiva (aun manteniendo dicha relación). pue- 
de crear problemas en cuanto a la calidad del clínker y el posible cumplimiento de la normativa vigente para cementos.

No obstante, las tolerancias son relativamente amplias en lo que se refiere al azufre que puede ser incorporado con el combustible, si se tratan crudos normales en el proceso.

Los cloruros, por su gran volatilidad y dificultad de depuración, crean serios problemas en el proceso y, por lo tanto, obligan a límites muy severos en cuanto a su aportación por parte de crudos y, especialmente, de los carbones empleados como combustibles.

En todos los casos, por la existencia de estos compuestos se crean acumulaciones o depósitos en zonas de la instalación por debajo de $1.100^{\circ} \mathrm{C}$, difíciles de evitar pero controlables en cierto modo.

En lo que a contaminación atmosférica se refiere, los modernos hornos de cemento, independientemente del tipo de combustible, son verdaderos "depuradores" o "trampas" para los compuestos gaseosos del azufre. Los óxidos de nitrógeno, siempre presentes en baja proporción, reducen su contenido en los gases al emplear carbón como combustible, según se ha demostrado en la experiencia industrial.

La emisión de polvo es prácticamente independiente del combustible empleado y las instalaciones y la normativa vigente hacen que se mantenga dentro de límites muy estrictos.

\section{BIBLIOGRAFIA}

(1) S. Sprung y W. Rechenherg (1978): "Las reacciones del plomo y del zinc durante la cocción del clínker de cemento”. Zement-Kalk-Gips, 31, n. $7,327$.

(2) E. Meintrup (1980): "El comportamiento del cloro en los hornos rotatorios con precalentador". Polysius Rev. 58, Polysius A. G. Beckun.

(3) R. Bucchi (1981): “Aspectos sobre el papel de los componentes minoritarios en el clinker de cemento". W. Cem. Technology, 12, 210 y 258.

(4) W. C. Dannenbrick, F. I. KChancwski, P. D. Hess y E. T. Losin (1977): "Propiedades del carbón y su influencia en el comportamiento de los sistemas de hornos con precalentador de ciclones" ZementKalk-Gips, 30, n.” 12, 618.

(5) E. Steinbiss (1979): "Experiencias con la precalcinación teniendo en cuenta la sustitución de combustible. Zement-Kalk-Gips, 32, n. 5 , 211.

(6) F. W. Locher, S. Sprung y D. OpItz (1971): "Reacciones en la atmósfera gaseosa del horno". Generalbericht Fachbereicht 4, VDZ-Kongress 71, 149.

(7) F. MatouscheK (1972): "Contribución para explicar la formación de grumos en el cemento". ZementKalk-Gips, 25, 395.

(8) C. Goes (1961): “Sobre el comportamiento de los alcalis en la cocción del cemento". Schr. Zementind. H. 24, Beton-Verlag, Düsseldorf.

(9) O. Kallauner (1970): "Sobre los álcalis en el cemento portland y su influencia en las propiedades del mortero y del hormigón". Baustoffind. 13, 381.

(10) H. Woors (1942): “Eliminación de álcalis por calentamiento con adiciones". Rock Products, $45,66$.

111) V. M. Hlonakaya y V. M. Stepanov (1969): "Sistemas para disminuir la influencia nociva de los álcalis en la calidad del clinker". Tsement (URSS), 4, 11.

(12) C. Froger y J. Stratmann (1961): "Tensión de vapor y de descomposición de algunas combinaciones alcalinas que participan en la fusión del vidrio". Glastechnik, Ber., 34, 311.

(13) S. SpRunc (1964): “Comportamiento del azufre durante la cocción del clinker de cemento". Schriftenreihe der Zement Industrie H. 31. Tonindustric Ztg. 89, (1965), 124.

14) H. Ritzmann (1971): “Circuitos de azufre en el sistema del horno rotatorio", Verfahrenstechnik der Herstellung von Zement. VDZ-Kongress 71, 1967.

(15) H. Hatano (1971): “Comportamiento del azufre en el horno con intercambiador de calor". Verfahrenstechnik der Herstellung von Zement. VDZ-Kongress 71, 169. 
(16) B. Osbaeck (1979): "Influencia de los álcalis en el comportamieno resistente del cemento portland". "Zement-Kalk-Gips, 32, 72.

(17) R. Bucchi (1980): "Influencia de la naturaleza y de la preparación de las materias primas sobre la reactividad de los crudos". 7." Congrès International de la Chimie des Ciments, I 1/3.

(18) H. M. Garret (1980): "Carbón e industria del cemento". Min. Engineering, 32, 188.

(19) S. Sprung (1966): "Composición quimica y mineralógica del polvo de los hornos de cemento". Tonindustrie Ztg. 90, 441.

(20) H. R. Norbon (1974): "Horno de via seca o de vía húmeda para su instalación". Rock Products, 77, mayo, 92 .

(21) S. SpRung (1981): "El efecto del procedimieno de cocción sobre la formación y las propiedades del clínker". 7..$^{\circ}$ Congrès International de la Chimie des Ciments, I-2/1.

(22) R. Bucchi y J. P. Meric (1981): "Influencia de las materias primas, de los combustibles y de los procedimientos de fabricación sobre la estructura y las propiedades de los clínkeres". 7." Congrès International de la Chimie des Ciments, IV, 37.

(23) S. Sprung y H. M. von Seebach (1968): "Contenido de fluor y emisión de fluor en los hornos de cemento". Zement-Kalk-Gips, 21, 1.

(24) H. M. Silla (1977): "Formación de costras o revestimientos mediante la fusión de sales". Zementkalk-Gips, 30, 487.

(25) C. SChmitr y C. Alter (1980): "El talio en el proceso de obtención del clinker de cemento portland". Bericht anlässlich der Sitzung der CETIC-Kommissionen Technik und Chemie. Dyckerhoff Zementwerk A. G. Wiesbaden.

(26) H. Ritzmann (1971): "Circuitos en los sistemas de horno rotatorio". Zement-Kalk-Gips", 24, 338.

(27) R. B. Engdahl y R. E. Barret (1977): "Polución del aire" 3." Edic. A. C. Stern., Vol. IV, 479.

(28) R. J. HILovsky (1977): "EPA Emmission Inventory/Factor Workahop, Baleigh (N.C.) septiembre 1977, p. 13-15.

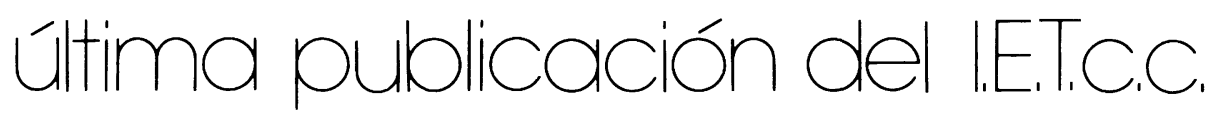

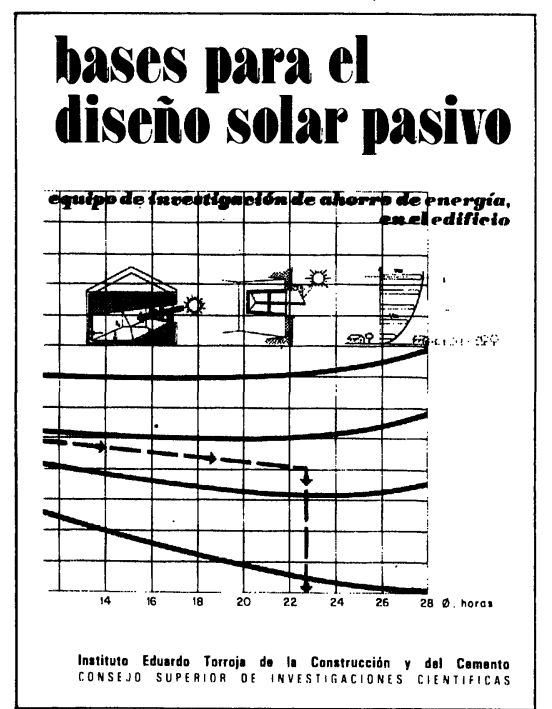

80
Equipo de Ahorro de Energia en el edificio

Dirección y coordinación:

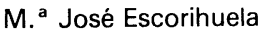
José Luis Esteban José Miguel Frutos Manuel Olaya Bernardo Torroja Arturo Garcia Arroyo

Las dificultades de suministro y el alto coste de los productos energéticos convencionales han despertado la atención de los usuarios, técnicos e industriales de la edificación hacia los procedimientos y sistemas en que se basa el aprovechamiento de otras fuentes alternativas de energia, principalmente la solar. Esto ha generado un rápido desarrollo industrial $y$ ha generado un rapido desarro lo industrial comercial qu este libro, arrastran los siguientes defectos: un mimético tecnologismo respecto de los sistemas convencionales que violenta las pe culiaridades de la energia solar (baja densidad y variabilidad en el tiempo), y una escasa selectividad en la aplicación de los sistemas y procedimientos pasivos dando origen a un ecumenismo arquitectónico solar, al margen de las condiciones climáticas y funcionales específicas de cada caso y lugar.

En este libro, utilizando criterios y metodologia pedagógicos, se dan los fundamentos e instru mentos teórico-prácticos necesarios para el planteamiento de tedo proyecto arquitectónico solar pasivo, de acuerdo con los principios éticos y económicos de conservación y ahorro de energía. Es decir: respeto de los presupuestos bioclimáticos, búsqueda de la máxima captación y acumulación de la radiación solar, y esmero en el aislamiento térmico de los cerramientos.

Un volumen encuadernado en cartulina ibiza plastificada, a cinco colores, de $16 \times 23 \mathrm{~cm}$, compuesto de 216 páginas, 217 figuras, 87 gráficos, 19 tablas y 10 cuadros.

Madrid, 1983. Precios: España 2.100 ptas.; 30 \$ USA 